03

\title{
Зависимости оптических свойств металлических наночастиц, размещенных в различных средах, от их температуры
}

\author{
(C) В.К. Пустовалов ${ }^{1}$, Л.Г. Астафьева 2 Ф \\ ${ }^{1}$ Белорусский национальный технический университет, \\ 220013 Минск, Республика Беларусь \\ ${ }^{2}$ Институт ффизики им. Б.И. Степанова НАН Беларуси, \\ 220072 Минск, Республика Беларусь \\ ฯ e-mail: astafev@dragon.bas-net.by
}

Поступила в редакцию 02.11.2020 г.

В окончательной редакции 10.11.2020 г.

Принята к публикации 26.11.2020 г.

Теоретически рассчитаны и исследованы зависимости факторов эффективности поглощения, рассеяния и ослабления излучения сферическими золотыми наночастицами с радиусами $r_{0}=10-125 \mathrm{~nm}$ в диапазоне длин волн $400-3000 \mathrm{~nm}$ и вольфрамовыми наночастицами с $r_{0}=50-125 \mathrm{~nm}$ в диапазоне длин волн 400-2500 nm. В качестве окружающих сред были выбраны вода, плавленый кварц, окись алюминия. Исследовано влияние изменения температур наночастиц и окружающей среды в широких диапазонах на оптические факторы рассеяния, ослабления и особенно поглощения излучения наночастицами. Установлено значительное изменение оптических параметров наночастицы при изменении ее температуры (включая ее плавление) и температуры окружающей среды, которое может оказывать определяющее влияние на динамику нагрева наночастицы и последующие тепловые процессы.

Ключевые слова: наночастицы, оптические свойства, температура, излучение, анализ.

DOI: $10.21883 /$ OS.2021.03.50657.271-20

\section{Введение}

В последние годы в многочисленных работах были исследованы оптические свойства металлических наночастиц (НЧ) [1-5]. Поверхностный плазмонный резонанс излучения на НЧ определяет ее оптические свойства и эффективность поглощения и рассеяния излучения НЧ. При этом, как правило, для определения оптических свойств НЧ использовались постоянные значения оптических параметров материалов НЧ при начальной температуре.

Процессы взаимодействия интенсивного лазерного и оптического излучений с НЧ представляют значительный интерес для различных применений в высокотемпературных лазерных нанотехнологиях и наномедицине, в солнечной наноэнергетике [6-12]. Интенсивное излучение поглощается НЧ, что приводит к нагреву НЧ до высоких температур, которые нас будет интересовать в первую очередь. С другой стороны, оптические параметры материалов НЧ, такие как показатели оптического поглощения и преломления, зависят от температуры. Следовательно, учет данной зависимости может приводить к изменению поглощения и рассеяния излучения НЧ по сравнению с этими параметрами при начальной температуре, к изменению динамики ее нагрева и результатов воздействия излучения на НЧ и окружающую среду.

В первую очередь поглощение лазерного излучения НЧ используется для их нагрева, изменения формы, размеров, структуры, оптических характеристик и других параметров НЧ. Особенно важным в технологическом смысле процессом взаимодействия лазерного излучения с НЧ является ее частичное или полное расплавление при нагреве за счет поглощенной энергии излучения. Экспериментальное исследование оптических и тепловых характеристик неравновесных процессов взаимодействия лазерного излучения с одиночной НЧ в различных средах с учетом ее нагрева, плавления и т.д. проведено в ряде работ [1-3]. При этом температура окружающей среды (воды) для коротких импульсов остается постоянной. Лазерная наномедицина активно использует нагрев НЧ для инициирования и реализации различных тепловых процессов в биотканях и лечения рака $[9,10]$. Для целей высокотемпературной нанофотоники исследование зависимости оптических характеристик НЧ от температуры также представляет значительный интерес. Разработка высокотемпературных наноструктурированных абсорберов оптического (солнечного) излучения сплошного диапазона предполагает их использование при высоких температурах нагрева НЧ и окружающих сред $[11,12]$. К сожалению, в литературе очень мало данных по индексам рефракции металлов и практически отсутствуют данные по оптическим свойствам НЧ при высоких температурах. Можно отметить только работу [13], в которой исследовано влияние частичного или полного расплавления НЧ под действием излучения на ее оптические характеристики. Существующий разрыв между опубликованными результатами [1-5] и потребностями в использовании НЧ 
при высоких температурах [6-12] требует исследований оптических свойств НЧ. Таким образом, исследование зависимости оптических свойств металлических НЧ от температуры представляет значительный научный и практический интерес.

Основной целью нашей работы является исследование на основе компьютерного моделирования зависимости оптических свойств НЧ от температуры в интервале $300-2000 \mathrm{~K}$ при действии интенсивного лазерного и оптического излучений.

\section{Результаты и обсуждение}

Рассмотрим оптические характеристики одиночной НЧ при лазерном или оптическом облучении, инициирующем неравновесные процессы нагрева НЧ. Фактор эффективности поглощения излучения НЧ $K_{\text {abs }}[14]$ определяет количество энергии, поглощенной НЧ, и влияние оптических показателей металла НЧ на процесс ее нагрева излучением. Значения фактора эффективности существенно изменяются при нагреве НЧ до высоких температур. Значение $K_{\mathrm{abs}}$ определяется значениями показателей преломления (рефракции) $n$ и поглощения $k$ материала НЧ. При этом данные показатели существенно изменяются при нагреве материала (металла) и фазовом переходе из твердого состояния материала (металла) в расплав. Значения показателей преломления и поглощения ряда металлов в твердом состоянии при начальной температуре $300 \mathrm{~K}$ приведены в $[15,16]$. Для некоторых диапазонов длин волн и температур, включая расплавленное состояние, значения этих показателей для определенных металлов приведены в [17-20].

При нагреве и плавлении происходит изменение оптических индексов рефракции и поглощения золота по сравнению с индексами при начальной температуpe [17-19]. Численные расчеты факторов эффективности поглощения $K_{\text {abs, }}$, рассеяния $K_{\text {sca }}$ и ослабления $K_{\text {ext }}$ различных сферических НЧ в широких спектральных диапазонах и при различных средах, окружающих НЧ, проведены на основе использования обобщенной теории Ми дифракции электромагнитного излучения на сфеpe $[14]$.

Рассмотрим оптические параметры золотой НЧ, размещенной в воде, при нагреве ее излучением. Был выбран спектральный диапазон 475-625 nm, в котором представлены зависимости показателей преломления и поглощения золота в широком диапазоне температур, включая температуру плавления [17]. Выбор этой области жестко обусловлен существующими экспериментальными данными [17] по оптическим параметрам в широком диапазоне температур. На рис. 1 представлены зависимости факторов эффективности поглощения $K_{\text {abs, }}$, рассеяния $K_{\text {sca }}$ и ослабления $K_{\text {ext }}$ излучения в диапазоне длин волн $\lambda=475-625 \mathrm{~nm}$ однородными твердыми (при температурах $\left.T_{0}=300,843,1336 \mathrm{~K}\right)$ и расплавленными (при $T_{0}=1336 \mathrm{~K}$, температура плавления золота) сферическими золотыми НЧ с радиусами $r_{0}=10,25$, $50,100 \mathrm{~nm}$, размещенными в воде при температуре $T_{\mathrm{M}}=300 \mathrm{~K}$. На рис. $1, a-i$ для твердой НЧ видны ярко выраженные максимумы факторов эффективности $K_{\text {abs, }}$, $K_{\text {sca }}$ и $K_{\text {ext }}$, обусловленные поверхностным плазмонным резонансом. С ростом $r_{0}$ от 10 до $25 \mathrm{~nm}$ значения $K_{\text {abs }}$ растут и затем начинают уменьшаться с $r_{0}=50 \mathrm{~nm}$. С другой стороны, значения факторов эффективности $K_{\text {sca }}$ и $K_{\text {ext }}$ растут с ростом $r_{0}$. Увеличение температуры приводит к уменьшению значений всех факторов для $\lambda<600 \mathrm{~nm}$ и некоторому их возрастанию для $\lambda>600 \mathrm{~nm}$. Для расплавленного состояния однородной золотой НЧ выраженный резонанс существует только для $r_{0}=50 \mathrm{~nm}$, при этом максимумы факторов эффективности $K_{\mathrm{abs}}, K_{\mathrm{sca}}$ и $K_{\text {ext }}$ выражены слабо.

На рис. 2 представлены зависимости фактора эффективности поглощения $K_{\text {abs }}$ излучения в диапазоне длин волн $\lambda=400-2000 \mathrm{~nm}$ для однородных сферических золотых НЧ с радиусами $r_{0}=10,25,50,100 \mathrm{~nm}$ при температурах $T_{0}=300,1173 \mathrm{~K}$, размещенных в воде при начальной температуре $T_{\mathrm{M}}=300 \mathrm{~K}$. Зависимости индексов рефракции и поглощения золота в приведенном диапазоне длин волн для указанных температур взяты из $[15,19]$. Данные результаты совпадают с представленными на рис. 1 в интервале $475-625 \mathrm{~nm}$, но представлены для более широкого диапазона длин волн. Эти результаты представляют интерес для воздействия коротких импульсов лазерного и оптического излучения на НЧ, размещенные в воде, без существенной передачи тепла от НЧ окружающей воде и без изменения ее фазового состояния. Это приближение справедливо для золотых НЧ с радиусами 30-100 nm при воздействии импульсов с длительностью $1 \mathrm{~ns}$ и для НЧ с радиусами 10-100 nm для более коротких импульсов.

В последнее время были предложены наноструктурированные твердотельные поглотители с поглощающими НЧ и прозрачной твердой матрицей для эффективного поглощения солнечной энергии, для применения в высокотемпературных технологиях, в качестве эмиттеров света для последующего переизлучения тепловой энергии и т.д. [20,21]. Под действием интенсивного оптического (солнечного) излучения на наноструктурированные твердые поглотители энергия излучения поглощается НЧ и после этого передается окружению за счет теплопроводности. Полезно использовать матричные твердые прозрачные материалы при $T_{\mathrm{M}}>10^{3} \mathrm{~K}$, такие как плавленый кварц, стекла, оксид алюминия, металлокерамика.

Оптические свойства наноструктурированных твердотельных абсорберов при их нагреве до температур порядка тысяч кельвинов непрерывным оптическим (солнечным) излучением меняются с температурой [20,21]. При использовании поглощающих НЧ в матрице солнечного абсорбера под действием солнечного излучения нагреваются до одинаковых температур и НЧ, и матрица. Следовательно, необходимо учитывать зависимости 


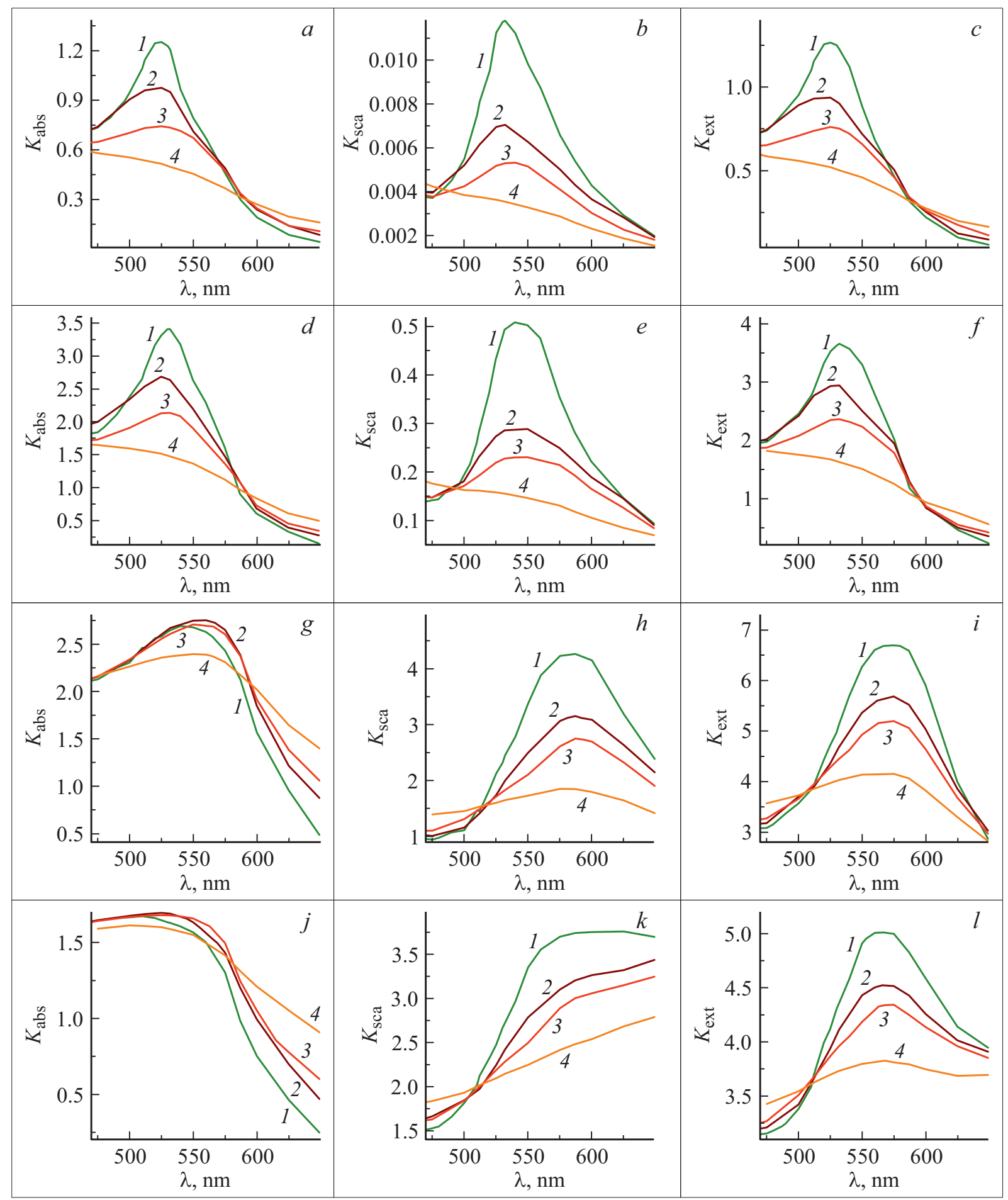

Рис. 1. Зависимости факторов эффективности поглощения $K_{\mathrm{abs}}(a, d, g, j)$, рассеяния $K_{\mathrm{sca}}(b, e, h, k)$ и ослабления $K_{\mathrm{ext}}(c, f, i, l)$ излучения в диапазоне длин волн $\lambda=475-625 \mathrm{~nm}$, однородными сферическими золотыми НЧ с радиусами $r_{0}=10(a, b, c)$, $25(d, e, f), 50(g, h, i), 100 \mathrm{~nm}(j, k, l)$ при температурах $T=273(1), 843$ (2),1336 (3, твердая), $1336 \mathrm{~K}(4$, расплав), размещенными в воде при $T=273 \mathrm{~K}$.

оптических параметров НЧ и матрицы от температуры. Для эффективного поглощения солнечного излучения с максимальной интенсивностью на длине волны $\lambda_{\max } \sim 550 \mathrm{~nm}$ можно использовать гомогенные метал- лические (Au, Ti, W, Ni и др.) НЧ. Важной особенностью интенсивного поглощения излучения НЧ и нагрева НЧ и окружающей среды является влияние температуры на эффективность поглощения излучения абсорбером и его 


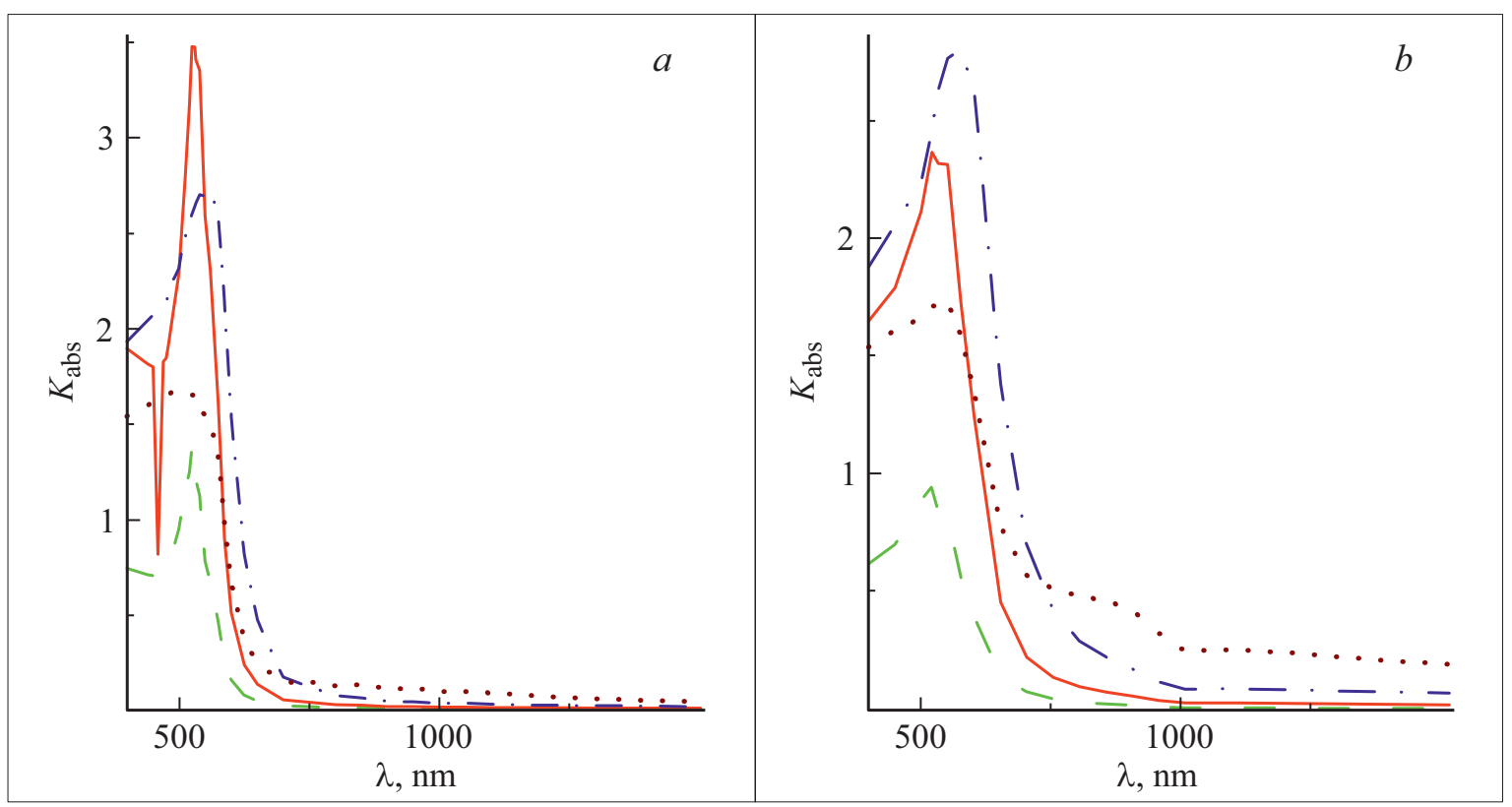

Рис. 2. Зависимости факторов эффективности поглощения $K_{\text {abs }}$ излучения в диапазоне длин волн $\lambda=400-1500 \mathrm{~nm}$ однородными сферическими золотыми НЧ с радиусами $r_{0}=10$ (штриховая зеленая кривая), 25 (сплошная красная кривая), 50 (штрихпунктирная синяя кривая), $100 \mathrm{~nm}$ (пунктирная коричневая кривая) при температурах $T=300(a), 1173 \mathrm{~K}(b)$, размещенными в воде при $T=273 \mathrm{~K}$.

последующий нагрев. Температуры плавления НЧ или материалов матрицы определяют максимальную температуру нагрева поглотителя интенсивным оптическим излучением.

На рис. 3 представлены зависимости факторов эффективности поглощения $K_{\mathrm{abs}}$, рассеяния $K_{\mathrm{sca}}$ и ослабления $K_{\mathrm{ex}} \mathrm{t}$ излучения в диапазоне длин волн $\lambda=300-2500 \mathrm{~nm}$ однородными сферическими золотыми НЧ с радиусами $r_{0}=50,75,100,125 \mathrm{~nm}$, размещенными в плавленом кварце, при одинаковых температурах НЧ $T_{0}$ и кварца $T_{\mathrm{M}}$, равных $300,1173 \mathrm{~K}$. Оптические постоянные кварца взяты из [22,23]. Представленные зависимости поглощения $K_{\mathrm{abs}}$, рассеяния $K_{\text {sca }}$ и ослабления $K_{\text {ext }}$ для двух значений температур 300 и $1173 \mathrm{~K}$ имеют ряд общих особенностей для всех значений радиусов $r_{0}$. Увеличение температур приводит к росту поглощения до $15 \%$ интегрально по спектру по сравнению с начальной температурой и уменьшению рассеяния во всем представленном интервале длин волн. При этом ослабление излучения НЧ примерно остается постоянным.

На рис. 4 представлены зависимости факторов эффективности поглощения $K_{\mathrm{abs}}$ излучения в диапазоне длин волн $\lambda=300-3000 \mathrm{~nm}$ однородными сферическими вольфрамовыми НЧ с радиусами $r_{0}=50,100$, $150 \mathrm{~nm}$, размещенными в окиси алюминия $\mathrm{Al}_{2} \mathrm{O}_{3}$, при температурах НЧ и окиси $T_{0}=T_{\mathrm{M}}=300,2300 \mathrm{~K}$. Нормированная интенсивность солнечного излучения $I_{\mathrm{sn}}$ также представлена на рисунке. Отметим, что исследование оптических свойств вольфрамовых НЧ, размещенных в окиси алюминия, при начальной температуре проведено в [21]. Оптические постоянные вольфрама и окиси алюминия взяты из [19,23].

Для $r_{0}=50 \mathrm{~nm}$ влияние температуры $T$ на $K_{\mathrm{abs}}(\lambda)$ невелико. При этом зависимость $K_{\mathrm{abs}}(\lambda)$ близка в зависимости $I_{\mathrm{sn}}(\lambda)$, что свидетельствует о возможности использования вольфрамовых НЧ в окиси алюминия для эффективного поглощения энергии солнечного излучения. Для НЧ с $r_{0}=100 \mathrm{~nm}$ и тем более с $r_{0}=150 \mathrm{~nm}$ смещения зависимостей $K_{\mathrm{abs}}(\lambda)$ для $T=2300 \mathrm{~K}$ по сравнению с $T=300 \mathrm{~K}$ являются существенными. При этом значения $K_{\mathrm{abs}}(\lambda, T=300 \mathrm{~K})>K_{\mathrm{abs}}(\lambda, T=2300 \mathrm{~K})$ для $\quad \lambda<1500 \mathrm{~nm}, \quad$ однако $\quad K_{\mathrm{abs}}(\lambda, T=300 \mathrm{~K})<$ $<K_{\mathrm{abs}}(\lambda, T=2300 \mathrm{~K}) \quad$ для $\quad \lambda>1500 \mathrm{~nm} . \quad$ НЧ $r_{0}=100 \mathrm{~nm}$ можно использовать для эффективного поглощения энергии солнечного излучения, поскольку интеграл $\int_{\lambda_{1}}^{\lambda_{2}} I_{s n}(\lambda) K_{\mathrm{abs}}(\lambda) d \lambda, \quad$ определяющий $\quad$ нагрев НЧ [24], будет сравним по величине с аналогичным интегралом для $r_{0}=50 \mathrm{~nm}$. Однако эффективность поглощения солнечной энергии НЧ с $r_{0}=150 \mathrm{~nm}$ существенно меньше в сравнении с $r_{0}=50,100 \mathrm{~nm}$ вследствие сдвига максимумов $K_{\mathrm{abs}}$ в инфракрасную область, где значения $I_{\mathrm{sn}}$ малы. Данные результаты представляют интерес для воздействия непрерывного оптического (солнечного) излучения на НЧ, размещенные в конденсированных средах, при существенной передаче тепла от НЧ окружающей среде и при равенстве температур НЧ и среды. 


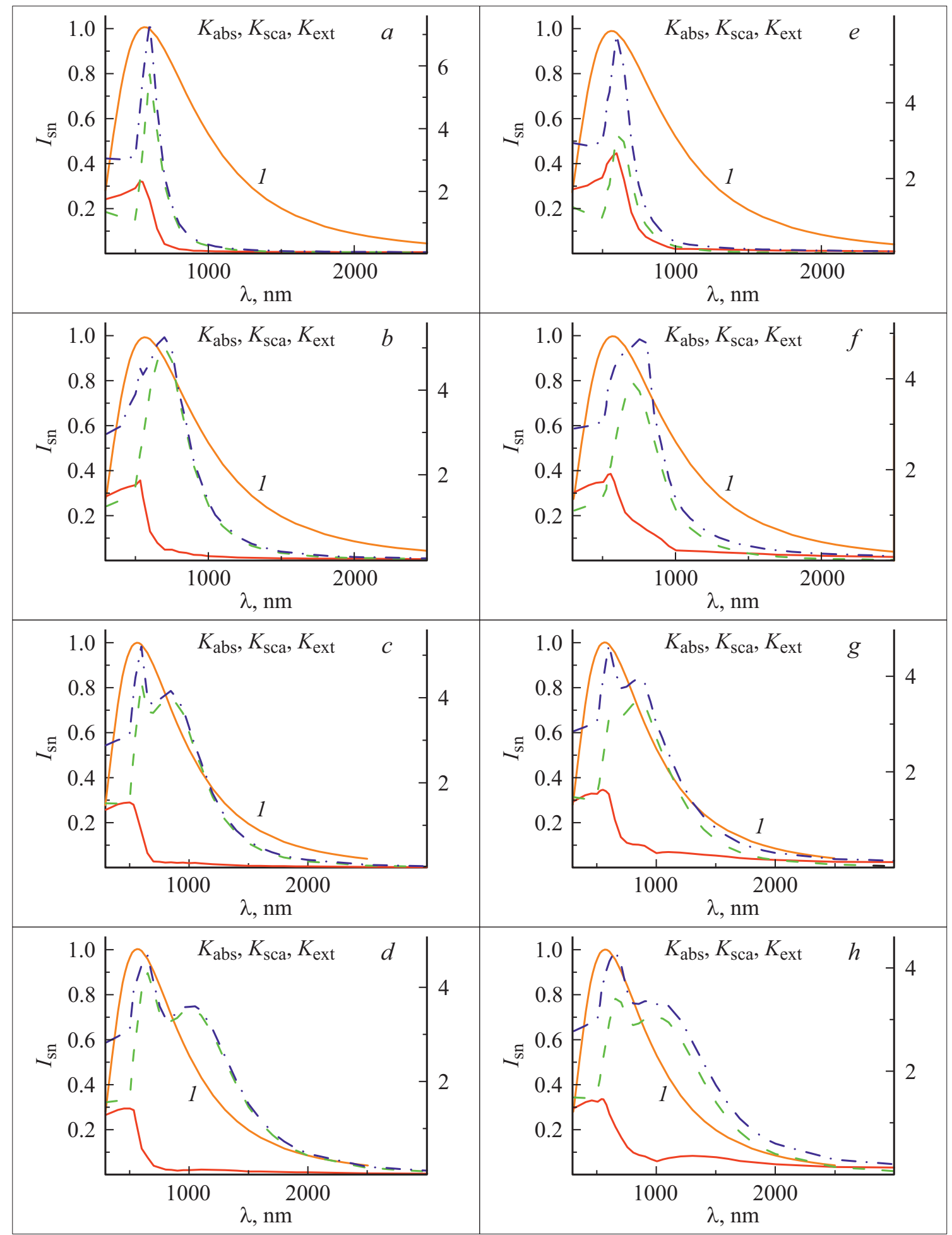

Рис. 3. Зависимости факторов эффективности поглощения $K_{\mathrm{abs}}$ (сплошная красная кривая), рассеяния $K_{\text {sca }}$ (штриховая зеленая кривая) и ослабления $K_{\text {ext }}$ (штрихпунктирная синяя кривая) излучения в диапазоне длин волн $\lambda=300-3000 \mathrm{~nm}$ однородными сферическими золотыми НЧ с радиусами $r_{0}=50(a, e), 75(b, f), 100(c, g), 125 \mathrm{~nm}(d, h)$, размещенными в плавленом кварце, при температурах НЧ и среды $T_{0}=T_{\mathrm{M}}=300(a, b, c, d), 1173 \mathrm{~K}(e, f, g, h)$. Нормированная интенсивность солнечного излучения $I_{\mathrm{sn}}$ (сплошная оранжевая кривая 1). 

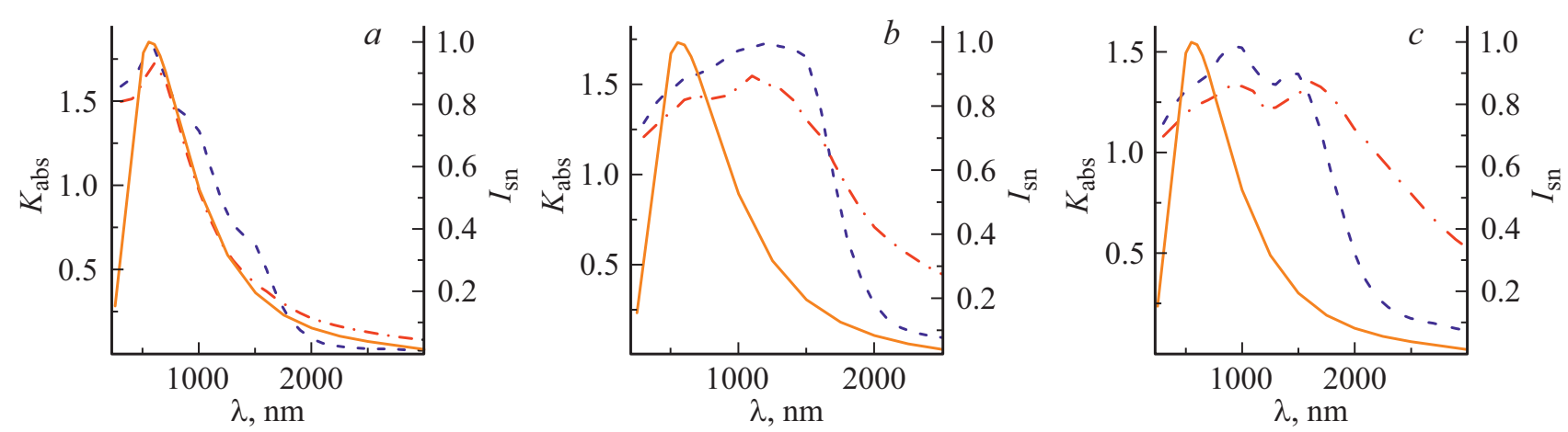

Рис. 4. Зависимости факторов эффективности поглощения $K_{\text {abs }}$ излучения в диапазоне длин волн $\lambda=300-2500 \mathrm{~nm}$ однородными сферическими вольфрамовыми НЧ с радиусами $r_{0}=50(a), 100(b), 150 \mathrm{~nm}(c)$, размещенными в окиси алюминия при температурах НЧ и окиси 300 (штриховая синяя кривая), $2300 \mathrm{~K}$ (штрихпунктирная красная кривая). Нормированная интенсивность солнечного излучения $I_{\mathrm{sn}}-$ сплошная красная кривая.

\section{Заключение}

Интенсивное лазерное и оптическое излучения поглощаются НЧ в процессе взаимодействия с ними, что приводит к нагреву НЧ до высоких температур, приводящих к последующим процессам. В то же время показатели оптического поглощения и преломления материала НЧ зависят от температуры, и учет данной зависимости может приводить к изменению поглощения излучения НЧ в процессе ее нагрева, изменению динамики нагрева и результатов воздействия.

Рассчитаны и исследованы зависимости от температуры факторов эффективности поглощения, рассеяния и ослабления излучения сферическими золотыми и вольфрамовыми НЧ, размещенными в воде, плавленом кварце и окиси алюминия соответственно. Исследования проведены в широких диапазонах изменения радиусов НЧ $r_{0}=10-125 \mathrm{~nm}$, длин волн $300-3000 \mathrm{~nm}$ и температур 300-2300 К. Установлено значительное изменение оптических параметров при изменении температуры НЧ (включая ее плавление) и окружающей среды, которое может оказывать существенное влияние на динамику нагрева НЧ и последующие тепловые процессы. В частности, уменьшение $K_{\text {abs }}$ в результате нагрева НЧ в течение действия импульса излучения может привести к необходимости увеличения интенсивности излучения для достижения плавления золотой частицы. Зависимости оптических свойств НЧ от ее температуры, их особенности при изменении параметров НЧ и окружающих сред представлены на рис. 1-4.

Использование результатов изучения взаимодействия интенсивного лазерного и оптического излучений с НЧ представляет значительный интерес для различных применений в высокотемпературных нанотехнологиях, лазерной наномедицине, солнечной наноэнергетике и высокотемпературной нанофотонике. Зависимость оптических параметров НЧ от температуры может оказывать существенное влияние на динамику и результаты процессов в данных нанотехнологиях.

\section{Финансирование работы}

Источники финансирования - Государственная программа научных исследований „Фотоника, опто- и микроэлектроника“, Институт физики НАН Беларуси, Белорусский национальный технический университет.

\section{Конфликт интересов}

Авторы заявляют, что у них нет конфликта интересов.

\section{Список литературы}

[1] Quinten M. Optical Properties of Nanoparticle Systems: Mie and Beyond. NY:: Wiley-VCH, 2011.

[2] Coronado E., Encina E., Stefani F. // Nanoscale. 2011. V. 3. N 12. P. 4042.

[3] Mayergoyz I. Plasmon Resonances in Nanoparticles. Singapore: World Scientific Publishing, 2013. 578 p.

[4] Trugler A. Optical Properties of Metallic Nanoparticles. Heidelberg: Springer, 2016. 156 p.

[5] Astafyeva L., Pustovalov V., Fritzsch W. // NanoStructures \& Nano-Objects. 2017. V. 12. N 10. P. 57.

[6] Nanomaterials. Processing and Characterization with Lasers / Ed. by Singh S., Zeng H., Guo C., Cai W. Wiley-VCH, 2014. $759 \mathrm{p}$.

[7] Stalmashonak A., Seifert G., Abdolvand A. Ultra-short Pulsed Laser Engineered Metal-glass Nanocomposites. Heidelberg, NY., Dordrecht, London: Springer, 2013. P. 27.

[8] Barcikowski S., Companigni G. // Phys. Chem. Chem. Phys. 2013. V. 15. N 8. P. 3022.

[9] Grosges T., Barchiesi D. // Molecules. 2018. V. 23. N 7. P. 1316.

[10] Bahadori A., Oddershede L.B., Bendix P.M. // Nano Research. 2017. V. 10. N 6. P. 2034.

[11] Masud M., Khan K., Chowdhury A., Sayeed Hassan N. Application of Thermo-fluid Processes in Energy Systems: Key Issues and Recent Developments for a Sustainable Future. Berlin: Springer, 2017. 457 p.

[12] Agrawal G., Khatri $R$. Solar thermal collectors: direct absorption using nanofluids: enhancing thermal performance by reducing heat losses and improving fluid properties. Lap Lambert Academic Publishing, Riga, 2016. 368 p. 
[13] Pustovalov V.K., Chumakov A.N. // J. Appl. Spectr. 2017. V. 84. N 1. C. 71

[14] Bohren C.F., Huffman D.R. Absorption and Scattering of Light by Small Particles. NY: Wiley, 1983. 661 p.

[15] Johnson P.B., Christy R.W. // Phys. Rev. B. 1972. V. 6. N 9. P. 4370.

[16] Refractive index database. http://refractiveindex.info/

[17] Otter M. // Z. Physik. 1961. V. 161. N 2. P. 163.

[18] Schmid M., Zehnder S., Schwaller P., Neuenschwander B., Held M., Hunzike U., Zurcher J. // Advanced Laser Technology, 2-6 September 2012, Thun, Switzerland.

[19] Aksyutov L.N. // J. Appl. Spectr. 1977. V. 26. N 5. P. 656.

[20] Kim J.U., Lee S., Kang S.J., Kim T. // Nanoscale. 2018. V. 10. N 12. P. 21555.

[21] Gao J.D., Zhao C.Y., Wang B.X. // J. Appl. Phys. 2017. V. 121. P. 113105.

[22] Wray J., Neu J. // JOSA. 1969. V. 59. P. 774.

[23] Лингарт Ю.К., Петров В.А., Тихонова Н.А. // ТВТ. 1982. T. 20. № 5. C. 872.

[24] Pustovalov V.K. // Springer Nature Appl. Sci. 2019. V. 1. N 356. 